fat. The edge of the liver was palpable one finger's breadth below the costal margin, but the spleen could not be felt. The urine was acid, its specific gravity was 1040 , it contained no albumin but a large quantity of sugar. The temperature was $98^{\circ} \mathrm{F}$., the pulse was 116 , and the respirations were 20 . The weight of the patient was $25 \mathrm{lb}$. From Sept. 22nd to 29 th no restrictions were made as to the diet, so that a correct idea of the natural tendency of the disease could be obtained. The child was allowed milk and.water ad libitum, bread-and-butter, meat, fish, potatoes, and rice pudding. It was soon evident that the disease was progressive. The child cried constantly for drink and passed urine very frequently (as much as 2500 cubic centimetres in 24 hours), while the sugar reached 11 per cent. It is noteworthy that even at this time there was no enuresis, which is a not uncommon symptom. The patient would wake at frequent intervals at night and cry for attention and in consequence a very com. plete record of the amount of urine passed was obtained. On the 29th some changes were made in the patient's diet. All the bread was toasted and the potatoes and milkpuddings were discontinued. The distress was at once relieved, the thirst was less, the quantity of urine was diminished, and less sugar was excreted. This modified diet was continued until Oct. 8th and during this time the child lost $1 \mathrm{lb}$. in weight. The bowels were confined and enemata were required from time to time. A prolapse of the rectum of the size of a walnut came down occasionally but it was easily reduced. On this date strict diabetic diet was begun and the meals were as follow: for breakfast the child had bacon, egg, gluten bread in slices toasted and buttered, weak tea with cream and saccharin; for lunch she had Soy biscuits ; for dinner she had meat or fish, green vegetables, calf's-foot jelly, beef-tea, or milk; for tea she had egg lightly boiled, weak tea with cream and saccharin ; and for supper she had Soy biscuits with beef-tea. The effect was remarkable. The patient became quite comfortable and she ate, drank, and slept well. The intense thirst diminished, the quantity of urine fell at once to 1000 cubic centimetres in 24 hours, and in a few days fell again to from 500 to 600 cubic centimetres, while the sugar fell to 4 per cent. Several attempts were made to vary the food by substituting almond bread and biscuits and Soy for the gluten bread, but the child seemed perfectly contented, and, indeed, preferred the gluten bread, which after a time was her sole carbohydrate food. On the 24th fresh honey was added to the diet. The bulk of the sugar in honey is lævulose and it has been shown that this form of sugar has much less tendency to increase glycosuria than ordinary sugar. ${ }^{5}$ For the first two days the quantity of urine was slightly increased, 1250 cubic centimetres and 900 cubic centimetres being passed in the 24 hours, and the sugar reached 5 per cent. After that the quantity excreted fell to the former level. Drugs were then tried and arsenic (two minims of Fowler's solution three times a day) had a definite effect. The quantity of urine fell to about 500 cubic centimetres a day and the sugar dropped to 2.75 per cent. and on one occasion to 2.2 per cent. The weight, which bad fallen to $23 \frac{1}{2} \mathrm{lb}$., rose to $24 \frac{1}{4} \mathrm{lb}$. under the arsenic treatment. Unfortunately diarrhcea set in and the arsenic had to be stopped. Bismuth soon controlled the diarrhoa, and reliance was placed for a time upon diet alone. There is very little of interest to record until Jan. 7th, 1899, when the child was evidently not so well. Her appetite was poor, her face was flushed, and the temperature, which had hitherto been normal or subnormal, rose to $1028^{\circ}$, and she was restless and awake all night. The sugar fell to 2 per cent. On the 9 th the liver was found to be slightly enlarged and the face was unusually sallow. The evening temperature was $102^{\circ}$. On the 12th jaundice was noticed. The liver was palpable two fingers' breadth below the right costal margin. On the 13th there was still greater enlargement of the liver which was tender, and the jaundice was deeper. The temperature was $101^{\circ}$. The urine was bilestained and acid in reaction, its specific gravity was 1020 , and the sugar was 1 per cent. At the height of the jaundice the sugar fell to 0.7 per cent. and the specific gravity to 1016. During this time the quantity of urine passed was from 635 to 775 cubic centimetres per day. By the end of January the jaundice had quite disappeared and the liver had returned to its normal size. There was now

5 Solis Cohen: International Clinics, 1894, vol. iv, series 3 R. Saundby : ibid. Van Noorden: Twentieth Century' Medicine, vol, ii.
4 per cent. of sugar and the quantity of urine passed in the 24 hours reached from 900 to 1100 cubic centimetres. Codeine (quarter-grain doses) and tincture of opium (one-minim doses) were tried but failed to improve the results obtained by diet alone. During March the child appeared to be failing. She got gradually weaker and took less interest in her surroundings, but the weight never fell below $23 \frac{1}{4} \mathrm{lb}$. On the 24th the temperature rose to $1002^{\circ}$ and the pulse was 120 . Five days later the patient complained of sore-throat and examination of the fauces showed a small patch upon the left tonsil. A s rabbing was made and a pure culture of diphtheria bacilli was obtained. There was a little hoarseness, but at no time were there severe laryngeal symptoms. The temperature was $102^{\circ}$, the pulse was 156 , the respirations were 36 , and the sugar amounted to 3 per cent. There was no albuminuria. Acetone was present. On the 31st the child became drowsy and she died on the next day in coma. The excretion of urea while the patient was under observation varied from 3 to 4 per cent. There was no evidence of peripheral neuritis at any time. The knee-jerks were obtained with difficulty throughout. There were no pulmonary complications and the optjc fundi, which were examined regularly, were normal.

The necropsy was unfortunately incomplete, as an examination of the brain was not permitted, and owing to the delay in obtaining the sanction of the parents for the post-mortem examination the pancreas was in such a condition that minute changes were unrecognisable. There were, however, no gross signs of disease. The kidneys showed slight cloudy swelling, the wall of the urinary bladder was hypertrophied, while the liver and the bile-ducts presented no abnormality.

The question of diagnosis calls for no remark. As soon as the condition was thought of an examination of the urine made it certain. Heredity, which has been shown by an examination of the recorded cases to be a prominent feature in the diabetes of the young, played, so far as is known, no part in this instance. The effect of diet is well shown by the history of this case. The most interesting feature is, of course, the remarkable diminution of the sugar and the consequent drop in the specific gravity of the urine during: the attack of jaundice. It cannot be assumed that the obstruction of the bile-duct was the cause of this, for extensive cirrhotic changes in the liver are unaccompanied by glycosuria. It appears to be the result of the febrile state and it is observed in pneumonia and other conditions attended by pyrexia. The febrile state depends no doubt upon the presence of toxins in the blood, and these bodies in some unexplained way seem to prevent glycosuria. The incidence of the diphtheria undoubtedly precipitated the fatal coma as does the occurrence of other acute diseases in the diabetic. It is curious that the sugar did not disappear as it did in the previous pyrexial condition, but this may perhaps be due to the inability of the diphtheria toxin to diminish the formation of sugar. In this connexion it is important to note that. Hibbard and Marrisay ${ }^{6}$ have found that there is a transitory glycosuria in many of the severe cases of diphtheria, and that this condition of the urine is present in most of the fatal cases. It is often accompanied by albuminuria. Moreover the injection of antitoxin is occasionally followed for a few days by a slight glycosuria. The action of toxins and antitoxins upon the formation of sugar appears to be well worth experimental investigation.

Welbeck-street, W.

\section{OBSERVATIONS ON TUBERCULOUS AND} SYPHILITIC DISEASES OF THE EYE.

\section{BY LACHLAN GRANT, M.D., C.M. EDIN,}

SURGEON TO THE GESTO HOSPITAL, ISLE OF SKYE.

Mucir has lately been written about tuberculosis both as to its spread and as to its frequency in various parts of the country, rural and urban. A branch of this subject of some interest is the relative frequency with which tuberculosis affects certain of the organs of the body. During the paste few years while engaged in hospital and private practice I have taken notes of all the affections of the organ of vision which came under my observation and it is remarkable that amongst 2100 cases observed not one

\footnotetext{
6 Journal of Experimental Medicine, 1899, vol, iv., p. 137.
} 
was positively diagnosed as being directly due to the action of the tubercle bacillus. No cases of tuberculous disease of the conjunctiva, of tuberculous keratitis, of tuberculous iritis, of miliary tubercles of the choroid, or of inflammation of the optic nerves sometimes seen in patients affected with tuberculous meningitis were met with. Cases were observed to which the term "strumous" was applied, such as strumous blepharitis and strumous ulcers of the cornea. These terms, however, were applied in a general way as in other affections, meaning rather an inflammatory condition of the affected tissues occurring in individuals of weakly looking appearance and having an apparent predisposition to the development of the tubercle bacillus, but without the bacillus eausing, as far as was known, the actual inflammation present. Two cases of a doubtful tuberculous nature were noted. Both of them occurred in girls. In one of them the sclerotic was much injected and swollen at parts, especially anderneath the upper eyelid. This condition had lasted for 13 months and did not improve under the ordinary treatment for inflammation of the sclerotic. The other girl was affected with lupus vulgaris of the face and in the centre of her right cornea there had developed a peculiar, clear, bleblike elevation, which was thought might possibly be a lupoid affection of that tissue. But it may be said that none of the eases showed the characteristic sign of tuberculous infection which we meet with so frequently in other organs of the body-namely, progression, going on to disintegration of structures affected. This fact shows the great immunity which the eye enjoys from tuberculous disease and the strong natural resisting powers which its structures present to the invasion of the tubercle bacillus. This local power of resisting the attacks of the bacillus must be much more highly developed in the eye than in the majority of the other structures of the body. In the other departments of surgery we meet with tuberculons infections frequently, as diseases of glands, bones, and joints, and tuberculous disease of the larynx is not so very uncommon. In fact, diseases caused by the tubercle bacilli fill a good half of the beds on the surgical, and also many on the medical, side of all general hospitals.

Why, then, one may ask, is tuberculous disease of the eye so relatively rare? This is not an easy question to answer. We know that tubercle bacilli along with other microorganisms are present in the air in many places and that inhalation of the bacillus from the air is one of the usual modes of infection of tuberculosis in man, so that the bacillus will frequently come in contact with the external surface of the eye, landing in its conjunctival cul-de-sac. This local immunity, then, does not depend on the absence of the infecting agent. Again, the eye is one of the organs of the body most subject to traumata and inflammatory or catarrhal affections of its coats. Most of us can remember having suffered at some time or other from the presence of a foreign body or of having had a blow on the eye or conjuctivitis. Yet these various conditions, which no doubt lower the vitality of some of the tissues of the eye, do not predispose to the bacillus gaining a foothold and setting up local tuberculous disease. Here, also, the eye differs from many of the other organs of the body, for not infrequently tranmata and inflammatory conditions predispose to local tuberculous disease. For example, in tuberculous disease of the knee-joint the "inner condyle" is much more frequently the seat of tubercular disease than the outer, probably because it is more liable to strain," and Treves ${ }^{2}$ states that a "feature in tuberculosis is the trifling nature of the irritant or lesion, whereby the mischief may be in the first place localised." Then, in catarrhal affections of the respiratory mucous membranes in individuals affected with the tuberculous diathesis one is anxious lest pulmonary tuberculosis should follow. The rarity, then, of tuberculous eye disease cannot be due to the absence of local predisposing causes.

An important factor in preventing the growth of the bacillus when it has reached the eye is that the organ of vision is exposed to much bright sunlight, and Koch, as well as other observers, has demonstrated that the direct rays of sunlight are fatal to the bacillus of tubercle. Thus, any bacilli present should be rendered more or less harmless by the action of this important agent. Another agent much valued nowadays in the treatment of pulmonary taberculosis

1 Stoker on Experiences of Excision of the Knee-joint for Tubercular Disease, Practitioner, January, 1896.

2 Treves's System of Surgery, vol. i., p. 343. is fresh cold air and of this the eye gets plenty. The adjective "cold" has been used with air because the bacilli do not grow well with a low temperature, preferring a temperature of from $80^{\circ}$ to $120^{\circ} \mathrm{F}$. And, as Ransome has pointed out, a lowered temperature will probably have some action in hindering the growth of the bacilli or in making them less virulent. Recent sanatorium experiences at Nordrach point to cold and not artificially warmed fresh air as all important in the cure of tuberculous affections of the lungs. ${ }^{3}$ The organ of vision then is very favourably situated for the full play of these potent natural agents.

The comparative infrequency of tuberculous infections in the eye may also be partly due to the fact that the movements of the eyes and eyelids tend to dislodge the bacilli from their surfaces. Then the secretions, conjunctival and lacrymal, which are constantly being secreted and flowing away, will also help to remove the bacilli from the eye before they have had time to gain a foothold and multiply and to proceed to attack the contiguous structures. But in addition these secretions probably have some special action on the bacilli themselves, exerting an inhibitory action on their growth or rendering them more or less harmless. Thomson, Hewlett, and others in a series of experiments demonstrated that the allied nasal secretions have some such bactericidal action. Again, the surface tissues of the conjunctiva and cornea must present a formidable barrier to the advance of the bacilli. Does this resistance depend on any histological peculiarity of these tissues? It would seem so. The anterior epithelium of the cornea is composed of stratified nucleated cells of many layers and the conjunctival epithelium is largely composed of stratified prismatic epithelial cells. Now, Baumgarten has shown that if the epithelium covering the body, which is also largely composed of stratified cells, be inoculated in its superficial layers with tubercle bacilli local tuberculous disease does not result and that the bacilli require to be introduced through the skin into the subcutaneous tissues before tuberculous infection occurs. This may be due to the density of the stratified tissues or perhaps to the presence of something inimical to the growth of the bacilli in these cells.4 In lupus vulgaris, which is a form of tuberculosis attacking the skin, caused probably by the bacillus gaining an entrance through an abrasion, the bacillus when settled there does not have the same tendency to develop and cause disintegration of the structures affected as in the other parts of the bodyfor example, in the larynx or in the lungs. It seems as if the epithelial coverings of the eye had a similar, and perhaps somewhat greater, resisting power to the local development and spread of the tubercle bacillus.

An observer ${ }^{5}$ has stated that he was much impressed at the relatively small number of cases of pharyngeal tuberculosis as compared with laryngeal tuberculosis. As these affections are in some points analogous to, and in other points different from, eye tuberculosis it might be interesting to briefly compare them. The pharynx (like the eye) is protected by stratified epithelium and the larynx and bronchi by an epithelium which is not to the same extent stratified and which is surmounted by a row of ciliated epithelial cells. These latter cells are naturally more delicate and are not so easily replaced after, say, an inflammatory attack as ordinary epithelial cells. They do not thus present such a dense front to the bacilli. Such an epithelial covering, then, would be more liable to infection by the bacilli gaining a suitable landing-place. The fact, again, that the pharynx is frequently cleansed by gargles, coughing, and the rapid passage of infected material in swallowing ${ }^{6}$ will prevent the bacilli from remaining for any length of time on its surface. In the eye also I have shown how the bacilli are dislodged. The larynx, on the other hand, differs from the eye and pharynx in that it seems to act as a

3 It seems reasonable to suppose that the pathogenic bacteria-such as, for example, the influenza germs-will be kept more active and virulent when given off into hot than into cool atmospheres; in fact that so-called comfortable artificially heated places act as incubators for these microbes. This may to some extent account for the recent widespread epidemic amongst our legislators, as in a recent issue the British Medical Journal it was stated that the air in the House of Commons was too hot.

4 Should this be so it might be possible to obtain a bactericidal extract from these cells of one of the carnivorous animals more resistant to tuberculous infection for trial in tuberculosis. In cinomatous afiections also an extract of the skin of a young. cinomatous affections also an extract of the skin of a young pig migh be tried, as McFadyean states animal. (Practitioner, April, 1899.) September, 18:6, p. 136

, Clifford Allbutt's System of Medicine, vol, ii., p. 32. 
harbourer of the bacilli, especially its ventricles, ${ }^{7}$ and they cannot be so easily dislodged. Further, the laryngeal mucous membrane has not the great exposure of the eye to sunlight and fresh cold air. The absence of these two agents will no doubt have some influence in favouring the develop. ment of the bacilli in that region, as we know that sunlight is destructive to them and that they prefer a temperature of about the body heat to grow well, and such a temperature is present in the larynx. It is thus evident that the larynx is more favourably situated and constructed for the harbouring and for the consequent development of the infecting agent of tubercle than are the pharynx and the eye.

The organ of vision has still another line of defence, that which Metchnikoff and his followers believe to be the first line of defence in dealing with bacteria generally-namely, phagocytic destruction of the bacilli by the leucocytes and connective-tissue cells. Probably, also, the nutrient fluids circulating in the tissues of the eye may have some action in hindering the growth of the bacilli. So that if the bacillus, in spite of the adverse conditions to its advance already considered, enters the tissues through some lesion of the conjunctival or corneal epithelium it will reach the phazocytic cells in a more or less weakened condition and will thus be more liable to be devitalised by the action of these cells and fuids. While we thus see that the external surface of the eye is not very liable to tuberculous infection by the bacillus gaining an entrance from without the interior of the eye is sometimes affected with diseases due to the tubercle bacillus. If we inoculate the anterior chamber of the eyes of guineapigs, which animals seem very susceptible to tuberculous infection, local tuberculosis follows in about 10 days. In man, when internal tuberculous disease occurs, the bacilli probably reach the parts in the interior of the eye by way of the blood or lymph streams, this being evidence of that peculiar diathesis in which the tissues are only slightly resistant to the action of the bacilli, whereby the latter are enabled to gain the upper hand and so circulate in the chief vascular channels. But, as already mentioned, no cases of tuberculous iritis, miliary tubercles of the choroid, or optic neuritis, sometimes seen in cases of tuberculous meningitis, were observed, so that tuberculous diseases of the interior of the eye were equally uncommon in my series of cases.

It is evident, then, that the eye has good defensive arrangements against the onset of the bacilli of tubercle, presenting a natural and formidable barrier to their advance. As is frequently stated, the nidus is evidently unfavourable for their growth and so further multiplication and spread of the bacilli are arrested. Local resistance apparently plays an important rôle in determining the occurrence of tuberculous infection, as seen in the skin and in our organ of vision. It would appear as if these local defensive arrangements were almost of a perfect nature in our organ of sight. In fact, tuberculous infection of the eye seems to me to occur so rarely that the organ may be considered "naturally immune," thus resembling the pharynx, thyroid body, pancreas, and ovary. In marked contrast to this apparent immunity of the eye from tuberculous infection in my observations was the very great frequency with which it was affected by the virus of syphilis, which virus caused many serious affections of that organ. I traced in a consecutive series of 1272 cases 37 patients with diseases of the eye resulting from syphilis, congenital or acquired. These were made up of the following: interstitial keratitis, 19 cases; syphilitic iritis, nine cases and choroidal affections, nine cases. This number does not probably represent all the syphilitic cases, many more being saspected in patients having old corneal opacities, iritic adhesions, obscure choroidal inflammations, optic atrophy, and eye affections the result of intra-cranial diseases.

The eye seems to be one of the organs of the body which is most frequently attacked by syphilis. It is thus evident that the resisting powers of the eye to syphilis, as with the rest of the body, is practically nil. In trying to discover the reasons for this greater frequency of syphilitic diseases in the eye as comparsd with tuberculous affections in that organ it might be instructive to look into the nature of the two diseases and also into their various modes of producing infection. Syphilis is a chronic infectious disease, being thus similar to tuberculosis which, as a rule, is also slow in its progress. We know that the disease must be caused by a microorganism of a parasitic nature. At present, however, not much is known regarding its habits or nature. This micro-organism differs from the tuberculous one in that

? J. Horne: British Medical Association Meeting, Edinburgh, 1898. it is incapable of producing the disease by aerial infection, but it is far more contagious than the germ of tubercle syphilis being one of the most contagious of diseases. This has often been proved by frequent accidental inoculations from patients suffering from primary or secondary syphilis. ${ }^{8}$ The most minute quantities of blood from such a syphilitic subject have, or the serum secreted from an abrasion has, proved fully contagious and produced the complete disease in another person. This is not the case, to the same extent anyway, with the serum and blood of a tuberculous patient, and it is well known that secretions containing the infecting agent of tubercle frequently come in contact with the tissues of healthy persons and yet but rarely produce general tuberculous disease. Again, if we contrast the hereditary aspect of the two diseases we find that in syphilis the virus is transmitted by the parent to the offspring either at the date of conception-i e., by sperm or germ transmission-or at a later date, when the fotus may be infected through the placenta by the syphilitic virus. In the communication of this infecting agent by the spermatozoid or ovum it is mechanically attached to one or other of these bodies. ${ }^{9}$ This is rarely the case with the virus of tubercle, which in nearly all of the offspring of tuberculous parents is not transmitted to the child by the spermatozoid or ovum, but on]y a predis position to its development at some future time, the actual bacillus which is the cause of the disease being but on the rarest occasions transmitted. ${ }^{10}$ And Woodbead in an article on the Bacteriology of Tuberculosis states: 1l "Hereditary tuberculosis may be left out of account, for although there may be a few cases of congenital tuberculosis-i.e., of tuberculosis contracted in the womb and due to the passage of the tubercle bacillus from the mother or membranes to the child or animal-all statistics point to the fact that tuberculosis is a disease which is contracted after birth-a most comforting knowledge for families in whom tuberculosis has been rife."

From the foregoing it will be evident that when syphilitic infection occurs the virus does not tend to remain more or less localised like the tuberculous virus but invariably circulates throughout the whole system, even early in life, the virus finding the tissues of probably all non-syphilitic individuals a suitable nidus for its development, so that the infecting agent will easily reach the eye, where no doubt it will multiply and in some as yet unknown manner produce the various affections of the tissues of that organ which are classed as syphilitic. On the other hand, the blood of a tuberculous person does not seem in most cases to have the bacilli circulating in the same extensive way as the virus of syphilis. The tissues of many individuals seem to offer greater resistance to the onset of the tuberculous infecting agent, and general dissemination of the bacilli is seen more frequently when the local tuberculous disease has opened into a blood-vessel and thus allowed the circulation of the infecting agent. The bacilli therefore only comparatively rarely reach the internal eye tissues. This may to some extent help to demonstrate why syphilitic infections in the eye are so much more frequent as compared with tuberculous infections of that organ.

In concluding this paper it might be useful to consider the reason why the syphilitic virus does not give rise to the disease in healthy individuals by aerial infection, like tuberculosis. In the mouth-secretions of syphilitic patients the virus is often present and is frequently expectorated on to pavements and floors, so that when the sputum dries the virus will be liberated and, like the infecting agent in tuberculous sputa, get freely blown about and so come in contact with other people's tissues. It cannot, however, reach them in an actively infectious state The infecting agent is apparently rendered harmless whenever it has left the system and been exposed to nature's disinfectants, light and air. Perhaps the oxygen in the latter is the chief agent inimical to the development of the virus. ${ }^{12}$ The syphilitic virus does not appear to be a typical anaerobic organism, as it readily multiplies in the blood-stream which carries a large quantity of oxygen. Another interesting point to consider is why the

\section{Clifford Allbutt's System of Medicine, vol. li., p. 696. \\ Fdinburgh Medical Journal, April, 1898, p. 421.
10 Hamilton's Pathology, 1894, vol. i., p. 153. 11 Practitioner, June, 1898, p. 596 .}

12 One would suppose that the oxygen gas treatment of wounds infected by the poisons of syphilis and tetanus would be a useful adjunct to general treatment of these diseases. This may have been tried already, although it has not come under my notice. 
virus of syphilis is so easily conveyed by the spermatozoa and ova. May it not be, as has been stated, ${ }^{13}$ that the virus of syphilis "is a very small bacillus"? There is in addition to this the probability that the virus is possessed of some means by which it can mechanically attach itself to these body cells, such as by a glutinous secretion of its cell envelope or conceivably that it has minute spinous processes like some pollen grains; or, again, by its having flagella which would allow of its motility. By these means the bacillus would also more easily become attached to, or entangled with, other protoplasmic cells, such as the phagocytes, and if so it would also, in addition to allowing a general spread of the infecting agent, more readily permit of the so-called phagocytic action of these cells. Perhaps as well as being an exceedingly minute bacillus the syphilitic organism may bave a very delicate protecting cell envelope which would permit of its being more speedily affected by agents inimical to its growth, such as air, and the medicinal remedies-mercury and iodide of potassium. These hypotheses may to a certain degree account for the fact that syphilis is not such a fatal disease and the same hypotheses might hold good with some of the other pathogenic bacteria.

Isle of Skye.

\section{MUSCULAR HYPOTONIA IN EPILEPTICS.}

By GEORGE E. RENNIE, M.D., M.R C.P. LOND., IA'LE PHYSICIAN AND PATHOLOGIST TO TRE PRINCE ALFRED HOSPITAL, SYDNEY.

Is normal conditions the muscles are kept in a condition of slight tension which is maintained in whatever physiological state they may be in-i.e., whether at rest or relaxed or anting synergically with other muscles, and of course when contracting under the influence of the will. When a muscle-for example, a flexor of the elbow-is at rest the muscle fibres are kept tense, like a string kept taut between two fixed points. When the elbow is flexed by passive movement the flexor muscle, instead of being flabby and loose, is still kept taut although the muscle is taking no active share in effecting the flexion of the limb at the joint or in maintaining it in that position. So, too, we know that when a group of muscles contracts the muscles with opposing actions also contract slightly, so that they are not in a condition of relaxation but of slight tension. The advantage of this condition of tone is obvious since it enables muscular movements to he effected with a minimum initial expenditure of muscular energy. But this condition is maintained outside the sphere of our consciousness; it is quite involuntary on our part and as such must be maintained by some reflex influt nce exerted upon the muscle centres. We know that if the anterior cornual cells of the spinal cord or the motor nerves be diseased the corresponding muscles become flaccid, and if experimentally a motor nerve be cut the muscle at once elongates. This clearly indicates that some impression or stimulus has been passing to the muscle, and when the nerve is cut this stimulus ceases and the muscle relaxes. Similar muscular relaxation with intact cornual cells and motor nerves occurs if the posterior nerve roots be cut experimentally. Hence we must assume that muscle tone is, in part at any rate, maintained by reflex influences exerted upon the muscles along the motor nerves from the motor cells in muscle centres and excited by sensory stimuli arising from the periphery. If this be true then we should expect that any break in this reflex arc would be followed by a loss of tone in the muscle connected with that arc. Loss of muscle tone, or, as Frenkel has called it, "hypotonie musculaire," is now a well-recognised feature in the symptom-complex of tabes dorsalis. In tabes there is invariably disease of the posterior nerve roots with varying degrees of anæsthesia of the skin and the deeper structures and thus a loss of conduction of sensory impressions from the periphery to the motor nerve cells. This will explain, in part at any rate, the muscular bypotonia seen in this disease. though it is quite possible that the condition of lowered nutrition in the muscles has some share in its production. In tabes this hypotonia is met with in an extreme degree and more or less in all the muscles of the body, of the trunk as well as of the limbs. For example, in a case of tabes which $I$ saw

13 Hutchison, jun. : Treves's System of Surgery, vol. i., p. 135. recently in a woman, aged 30 years, an in-patient at the National Hospital for the Paralysed and Epileptic, Queensquare, under the care of Dr. Bastian, there was advanced optic atrophy and the patient could stoop down and place the palms of her hands flat upon the ground while keeping her knees fully extended without the least inconvenience. When in bed she could bend forwards so as to place her head on the bed between her legs. A similar condition of relaxation of muscles existed in the limbs, so that the degree of passive movement was only limited by the bony and ligamentons configuration of the joints. But while in tabes hypotonia appears generally to involve all the muscles it is not so in all cases of this disease; it may be a localised condition. I have already referred to the existence of bypotonia in Friedreich's ataxy-a disease analogous to tabes in the disease of the posterior nerve roots but in which there is not the same nutritional change in the muscles. In disease of the anterior cornual cells, as anterior poliomyelitis, or in disease of the motor nerves, there is of course paralysis of the corresponding muscles, and if these be all the muscles of a limb then the limb hangs flaccid and flail-like; here it is not possible to differentiate between the loss of tone and the actual palsy. We have seen, then, that muscular hypotonia can exist where there is disease of either the motor or sensory part of the reflex arc, and I wish now to draw attention to its existence in cases where there is reason to suppose that the break in the reflex arc occurs in the motor nerve cells in the cortex. Thus it occurs as an inter-paroxysmal condition in certain cases of epilepsy. I will briefly record three cases.

CASE 1.-A boy, aged 10 years, had been on several occasions an in-patient at the National Hospital for the Paralysed and Epileptic, Queen-square, under the care of Dr. Bastian. He had been suffering from epileptic fits for a number of years, and when admitted again recently he had a succession of fits each day. There was no evidence of any gross intracranial or spinal disease. Mentally he was very dull and stupid, and could not be got to answer questions. He was somewhat emotional, and was dirty in his habits. He bad had no fit for two days before I examined him and ascertained that the following passive movements (amongst others) could be performed easily without causing the slightest pain or inconvenience. His bead could be extended on the trunk so as to bring the occiput almost in contact with the spine ; it conld be flexed so as to bring the chin on to the sternum; it could be rotated and the chin placed on the top of either shoulder, and even further, so that he could look almost directly behind him. He could not bend the trunk sufficiently to place his head on the bed between his legs, nor could he touch his toes with his fingers while keeping his knees fully extended. In the lower extremities the leg could be flexed on the thigh so as to bring the heel against the buttock of the same side and also flexed still further so that the heel could be placed above the umbilicus. The leg could also be flexed and the foot placed on the outer side of the trochanter of the same side. Similar wide range of movements in the arms was observed, and when holding the forearm or the leg and shaking it the hand or foot, as the case may be, was seen to swing about in every direction as if it did not belong to him. But with this condition of relaxation of muscle there was also a slight degree of resistance on first attempting these passive movements, but this was readily overcome. This is interesting in view of the fact that in this case all the deep reflexes were well marked, knee-jerks, ankle-jerks, and even ankle-clonus being obtained. There was no actual paralysis and all voluntary movements were performed with a moderate amount of muscular power.

CASE 2.-A lad, aged 17 years, had been subject to epileptic fits for several years and when admitted into the National Hospital for the Paralysed and Epileptic under Dr. Bastian he was having one daily. He was extremely dull and stupid and was dirty in his habits. There was no evidence of any gross disease of the nervous system. The fits were bilateral and genuinely epileptic in character. In this case the trunk muscles did not show any marked degree of hypotonia, but the limb muscles were as flaccid almost as in the first case. In this case also there was some resistance on first attempting the movements, which, however, was easily overcome and the extreme passive movements were performed without the least degree of pain or discomfort All the deep reflexes were active and ank'e clonus was obtained.

CASE 3.-A boy, aged 11 years, had been subject to fits for three years before admission to hospital. The fits occurred generally every two or three days. There was no 